FRI0304

DEFINITION AND STANDARDIZATION OF INTERSTITIAL LUNG DISEASE ASSESSMENT BY ULTRASOUND: RESULTS FROM A DELPHI PROCESS AND WEBRELIABILITY EXERCISE BY THE OMERACT ULTRASOUND WORKING GROUP (WG)

Andrea Delle Sedie ${ }^{1}$, Lene Terslev ${ }^{2}$, George Bruyn ${ }^{3}$, Tomás Cazenave ${ }^{4}$ Stavros Chrysidis ${ }^{5}$, Mario Diaz ${ }^{6}$, Marco DI Carlo ${ }^{7}$, Marilena Frigato ${ }^{8}$, Luna Gargani ${ }^{9}$, Marwin Gutierrez ${ }^{10}$, Alojzija Hocevar ${ }^{11}$, Annamaria lagnocco ${ }^{12}$ Aaron Juche ${ }^{13}$, Peter Mand ${ }^{14}$, Esperanza Naredo ${ }^{15}$, Mohammed A. Mortada ${ }^{16}$ Carlos Pineda ${ }^{10}$, Francesco Porta ${ }^{17}$, Viviana Ravagnani ${ }^{8}$, Carlo Alberto Scirè ${ }^{18}$, Teodora Serban ${ }^{19}$, Kate Smith ${ }^{20}$, Maria Stoenoiu ${ }^{21}$, Marika Tardella ${ }^{7}$, Karina Torralba ${ }^{22}$, Richard Wakefield ${ }^{20}$, Maria-Antonietta D'agostino ${ }^{23}$, OMERACT US Task Force on Lung US in Rheumatology. ${ }^{1}$ University of Pisa, Pisa, Italy; ${ }^{2}$ Rigshospitalet-Glostrup, Copenhagen, Denmark; ${ }^{3}$ Lelystad, Lelystad, Netherlands; ${ }^{4}$ Instituto de Rehabilitacion Psicofisica, Buenos Aires, Argentina; ${ }^{5}$ Esbjerg, Esbjerg, Denmark; ${ }^{6}$ Fundacion Santa Fe, Bogotà, Colombia; ${ }^{7}$ Università Politecnica delle Marche, Jesi, Italy; ${ }^{8}$ A.S.S.T Mantova, Mantova, Italy; ${ }^{9}$ National Research Council, Pisa, Italy, ${ }^{10}$ Instituto Nacional de Rehabilitación, Mexico City, Mexico; ${ }^{11}$ UMC, Ljubljana, Slovenia; ${ }^{12}$ Università degli Studi di Torino, Torino, Italy; ${ }^{13}$ Immanuel-Krankenhaus, Berlin, Germany; ${ }^{14}$ Medical University of Vienna, Vienna, Austria; ${ }^{15}$ Fundación Jiménez Díaz, Madrid, Spain; ${ }^{16}$ Zagazig University, Zagazig, Egypt, ${ }^{17}$ Pistoia, Pistoia, Italy, ${ }^{18}$ University of Ferrara, Ferrara, Italy,

${ }^{19}$ Ospedale La Colletta - ASL3, Genoa, Italy, ${ }^{20}$ NIHR Leeds Biomedical Research Centre, Leeds, United Kingdom; ${ }^{21}$ Université catholique de Louvain, Bruxelles, Belgium; ${ }^{22}$ Loma Linda University, Loma Linda, United States of America;

${ }^{23}$ Université Paris Ouest-Versailles-Saint Quentin en Yvelines, Paris, France

Background: Interstitial lung disease (ILD) evaluation is challenging, given the low sensitivity of X-ray and pulmonary function tests, and limited accessibility and radiation linked to repetitive HRCT. Lung Ultrasound (US) has shown potential in the evaluation of ILD of autoimmune diseases including systemic sclerosis.

Objectives: To define and asses the reliability of definitions of USdetected findings in ILD.

Methods: A taskforce (TF) within the OMERACT US WG performed a literature review (LR) to identify US lesions in ILD, a Delphi exercise to define these lesions and a web-based exercise to test the reliability of these definitions by using either intraclass correlation coefficient (ICC) or kappa statistics. Prior to the Delphi exercise all participants received training files and were subsequently asked to provide clips on BL, PLI and normal findings. Based on the results of the LR, which identified B-lines $(\mathrm{BL})$ and pleural line irregularity (PLI) as main US findings in ILD, the Delphi exercise contained statements on US definitions on BL and PLI. After reaching agreement (>75\%) on the above mentioned items, a set of 80 high-quality clips ( 30 for $\mathrm{PLI}, 50$ for $\mathrm{BL}$ ), was selected and distributed to the TF members in order to score them (semiquantitatively, 0-2 for $\mathrm{PLI}$ and total number for $\mathrm{BL}$ )

Results: The 3 Delphi rounds and the web-based exercise were completed by 24 and 22 sonographers, respectively. Final definitions are reported in Table I. The web-based exercise showed moderate interreader reliability for both $\mathrm{BL}(\mathrm{ICC}=0.61)$ and $\mathrm{PLI}(\mathrm{kappa}=0.69)$.

Table I: definitions of PLI and BL

Pleural line irregularity (PLI): a loss of regularity that may be associated with an increase in thickness, focal, diffuse, or nodular

B-line (BL): a vertical hyperechoic reverberation artifacts that arise from the pleural line, extend to the bottom of the screen without fading, and move synchronously with lung sliding

Conclusion: US is a candidate imaging modality to identify and monitor ILD in rheumatic diseases; US consensus based definitions of main US findings were defined and endorsed. Further development is planned for validating US as an outcome measurement instrument for ILD.

Disclosure of Interests: Andrea Delle Sedie Speakers bureau: Abbvie, UCB, Celgene, MSD, Lene Terslev Speakers bureau: Speakers fee from : Roche, Novartis, Pfizer, MSD, BMS, Celgene, George Bruyn : None declared, Tomás Cazenave: None declared, stavros chrysidis: None declared, Mario Diaz: None declared, Marco Di Carlo: None declared, Marilena Frigato: None declared, Luna Gargani: None declared, Marwin Gutierrez: None declared, ALOJZIJA HOCEVAR: None declared, Annamaria lagnocco: None declared, Aaron Juche: None declared, Peter Mandl: None declared, Esperanza Naredo Consultant for: Abbvie, Speakers bureau: AbbVie, Roche, Bristol-Myers Squibb, Pfizer, UCB, Lilly, Novartis, Janssen, and Celgene $\mathrm{GmbH}$, Mohammed A Mortada: None declared, Carlos Pineda: None declared, Francesco Porta: None declared, Viviana
Ravagnani: None declared, Carlo Alberto Scirè: None declared, Teodora Serban: None declared, Kate Smith: None declared, Maria Stoenoiu Grant/research support from: Abbvie, Roche, Wyeth, Marika Tardella: None declared, Karina Torralba: None declared, Richard Wakefield: None declared, Maria-Antonietta d'Agostino: None declared DOI: 10.1136/annrheumdis-2019-eular.7259

\section{FRI0305 1 PREDICTIVE VALUE OF INNATE LYMPHOID CELLS IN SYSTEMIC SCLEROSIS}

Alina Soare, Stefanie Weber, Markus Luber, Simon Rauber, Thomas Wohlfahrt, Georg Schett, Jörg Distler, Andreas Ramming. Friedrich-Alexander-University (FAU) Erlangen-Nürnberg and Universitätsklinikum Erlangen, Department of Internal Medicine 3 - Rheumatology and Immunology, Erlangen, Germany

Background: Systemic sclerosis (SSc) is a chronic autoimmune disease with a high morbidity and mortality. Activation of the immune system is a characteristic feature of SSc. Numerous studies have suggested that type 2 and type 3 cytokines are key drivers of progressive fibrosis. Recently, innate lymphoid cells (ILC) are emerging as an important cellular source of type 2 and type 3 cytokines triggering fibrotic tissue remodeling independently of the adaptive immune system. ILCs are characterized by the absence of conventional lineage markers. Similar to $T$ cells, they are categorized into three groups (ILC1, ILC2, ILC3), according to distinct pattern of cytokine production and the requirement of specific transcription factors guiding their development and function. Increased levels of ILC2 were found in patients with SSc. However, the contributive role of ILC2 and ILC3 in pathogenesis of SSc is not completely understood.

Objectives: We aim in our study to evaluate the predictive role of ILC2 and ILC3 in SSc patients.

Methods: We conducted an observational retrospective study on 52 patients with SSc fulfilling the 2013 ACR/EULAR classification criteria. Yearly clinical, laboratory and investigational data according to EUSTAR recommendations were collected. Blood samples collected between 15.09.2014 and 15.01.2015 were analyzed by flow cytometry and ILC2 and ILC3 counts were measured. The predictive value of ILC2 and ILC3 during a 2-year follow-up was analyzed using SPSS 21.0. ILC3 counts were also analyzed in skin sections (10 patients with SSc and 10 healthy controls) by immunofluorescence (IF) staining using two complementary panels of markers. Cytokine production of skin resident ILC3s was additionally analyzed by IF staining.

Results: 52 patients were included in the study, 78\% female, $63 \%$ limited cutaneous SSc with a mean follow-up time of $2.85 \pm 1.28$ years. At baseline we have shown that circulating ILC2s are significantly increased compared to gender and age-matched healthy controls. Increased numbers of ILC2s significantly correlated with worsening of mRSS calculated by five point increase in mRSS or $25 \%$ increase from baseline $(p<$ $0.001 ; 95 \% \mathrm{Cl} 1.39$ - 3.26). ILC2 counts also correlated with $5 \%$ decrease of diffusion capacity of carbon monoxide (DLCO) during the follow-up time $(p<0.0001 ; 95 \% \mathrm{Cl} 1.83-3.49)$. Worsening of forced vital capacity (FVC) assessed as $5 \%$ decrease over 2 years was also significantly correlated with an increased number of ILC2s ( $p<0.0001 ; 95 \%$ $\mathrm{Cl} 1.27$ - 3.04). In contrast, we did not find any correlation regarding increase in pulmonary arterial pressure assessed by echocardiography. Although new appearance of digital ulcers could not be predicted by ILC2 counts, increased numbers of ILC2s were correlated with digital ulcers at follow-up. ILC2s did not have a predictive value for death during the follow-up time. In contrast to ILC2, circulating ILC3s were not found to be correlated with worsening of disease activity. However, ILC3s were prominent in fibrotic skin of SSc patients compared to healthy controls, and showed strong production of IL-17.

Conclusion: Here, we provide first evidence for a role of ILC2S as potential prognostic marker of disease progression in SSc. Circulating ILC3 counts were not elevated in SSc. However, ILC3s showed strong cytokine production in the fibrotic skin of SSc patients. The functional impact has to be further evaluated.

Disclosure of Interests: Alina Soare: None declared, Stefanie Weber: None declared, Markus Luber: None declared, Simon Rauber: None declared, Thomas Wohlfahrt: None declared, Georg Schett: None declared, Jörg Distler: None declared, Andreas Ramming Grant/research support from: Novartis

DOI: 10.1136/annrheumdis-2019-eular.7639 